design. Among other cardiac risk factors and noncardiac comorbidities, the high-risk group had significantly worse left ventricular function and more nonelective operations ( $80 \%$ vs $28 \%)$ than the controls.

The study has two limitations. The first is incomplete followup, with $72 \%$ returning completed questionnaires. Although this is generally an acceptable rate of return, it is possible that nonresponders are different from responders, and this may have biased the results. Second, surgeons and cardiologists may exercise judicious patient selection over and above the information provided by the risk model, so our conclusion may not necessarily apply to all drastic-risk patients.

Although the EuroSCORE itself has been shown to be the most important single predictor of early outcome and resource use after heart surgery, ${ }^{2}$ we showed that it is possible to outperform the logistic EuroSCORE by an important margin. We believe that cardiac surgical centers should measure their own performance in the highest risk subsets before performing cardiac surgery in high-risk patients.

\section{Conclusions}

Our results suggest that despite substantial predicted and actual early mortality, long-term survival and good QOL make cardiac surgery worthwhile in selected high-risk patients. Predicted mortality greater than $50 \%$ alone should not be an absolute contraindication to surgery.

We thank Angela O'Farrell for her support with the Short-Form 36 Health Survey.

\section{References}

1. Michel P, Roques F, Nashef SA. Logistic or additive EuroSCORE for high-risk patients? Eur J Cardiothorac Surg. 2003;23:684-7.

2. Nilsson J, Algotsson L, Hoglund P, Luhrs C, Brandt J. EuroSCORE predicts intensive care unit stay and costs of open heart surgery. Ann Thorac Surg. 2004;78:1528-34.

\title{
Dissection of Kommerell's aneurysm mimicking aortic arch dissection on echocardiography: Multislice computed tomographic diagnosis
}

\author{
Tze-Yu Lee, MD, ${ }^{\text {a }}$ Shu-Hang Ng, MD, ${ }^{a}$ Chi-Di Liang, MD, ${ }^{\mathrm{b}}$ Ming-Jang Hsieh, MD, ${ }^{\mathrm{c}}$ and Sheung-Fat Ko, MD, \\ Kaohsiung, Taiwan
}

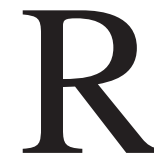

ight-sided aortic arch (RAA) is an uncommon anomaly that occurs in about $0.04 \%$ to $0.1 \%$ of the population. ${ }^{1}$ RAA is generally asymptomatic but can occasionally cause acute aortic symptoms because of dissection or aneurysmal rupture. ${ }^{1}$ Recent studies using imaging modalities, including transthoracic echocardiography (TTE), transesophageal echocardiography (TEE), multislice computed tomography (MSCT), or magnetic resonance imaging, have shown high accuracy in the diagnosis of acute aortic lesions. ${ }^{2-4}$ However, the diagnostic pitfalls of acute aortic diseases on echocardiography in patients with aortic arch anomalies have rarely been described. ${ }^{5}$ We report the MSCT diagnosis of a case of RAA associated with dissection of Kommerell's aneurysm (KA) mimicking aortic arch dissection on echocardiography.

\footnotetext{
From the Departments of Radiology, ${ }^{\mathrm{a}}$ Pediatric Cardiology, ${ }^{\mathrm{b}}$ and Cardiovascular and Thoracic Surgery, ${ }^{c}$ Chang Gung Memorial Hospital- Kaohsiung Medical Center, Chang Gung University, College of Medicine, Kaohsiung, Taiwan.

Received for publication June 23, 2006; revisions received July 14, 2006; accepted for publication Aug 8, 2006.

Address for reprints: Sheung-Fat Ko, MD, Department of Radiology, Chang Gung Memorial Hospital-Kaohsiung Medical Center, 123, Ta-Pei Road, NiaoSung Hsiang, Kaohsiung Hsien, 833, Taiwan (E-mail: sfa.ko@msa.hinet.net or sfatko@adm.cgmh.org.tw).

J Thorac Cardiovasc Surg 2006;132:1228-30

$0022-5223 / \$ 32.00$

Copyright $\odot 2006$ by The American Association for Thoracic Surgery doi:10.1016/j.jtcvs.2006.08.016
}

\section{Clinical Summary}

A 48-year-old man was admitted to the emergency department because of acute sharp chest pain that radiated to his back. He had a 10-year history of hypertension and hyperuricemia. Physical examination was unremarkable, except for increased blood pressure of 168/98 $\mathrm{mm} \mathrm{Hg}$. Electrocardiography revealed slight ST-T elevation in the V1 to V3 leads. Chest radiography showed a widened mediastinum with an RAA and right descending thoracic aorta. Emergency TTE showed an intimal flap in the widened aortic arch (Figure 1) and the descending thoracic aorta indicative of aortic dissection. Possible retrograde dissection to the ascending aorta was also suspected. Subsequent TEE revealed similar findings, but the ascending aorta still could not be definitively evaluated. Emergency surgical intervention was planned under the impression of type A aortic dissection. Before the operation, MSCT (4-detector-row, Volume Zoom; Siemens, Forchheim, Germany) demonstrated an RAA with a right descending aorta, a Kommerell's diverticulum with aneurysmal dilatation, and an aberrant left subclavian artery (ALSA; Figure 2). Dissection of the KA with intimal flaps was clearly demonstrated on axial and multiplanar reconstructed images, corresponding to the misdiagnosed aortic arch dissection on TTE and TEE. The entrance tear was identified at the KA. Extension of the intimal flaps to the ALSA, the right descending aorta, and the abdominal aorta was noted, with sparing of the RAA and ascending aorta, indicating type B aortic dissection. The patient refused surgical treatment of the KA and was conservatively managed with blood pressure and 


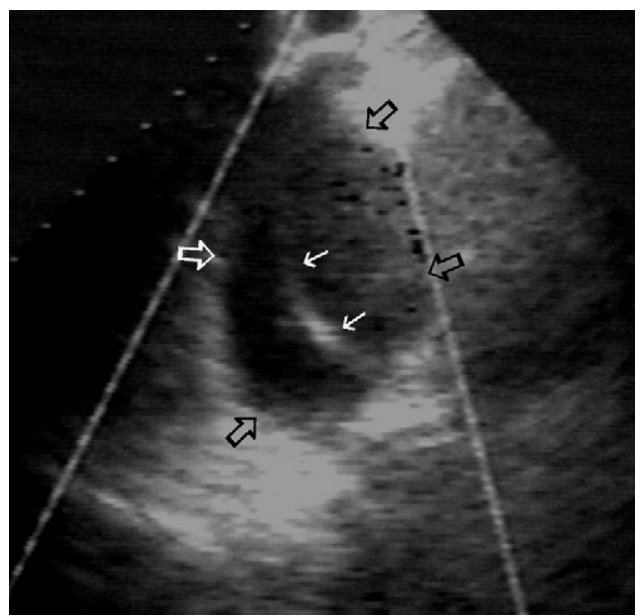

Figure 1. Transthoracic echocardiography shows an intimal flap in an aortic arch-like structure (open arrows), which is actually a Kommerell's diverticulum (arrows).

pain control. He was discharged 4 days later and remained in stable condition during regular follow-up for 3 years.

\section{Discussion}

Approximately $40 \%$ of RAA cases are associated with an aberrant subclavian artery (ASA) originating from a diverticulum, so-called Kommerell's diverticulum, which is formed by a remnant of the left dorsal aorta. ${ }^{1}$ Most patients with RAA are asymptomatic. However, RAA and aortic diverticulum can be confused with acquired aortic diseases, leading to unnecessary surgical intervention. ${ }^{1,5}$ Although rare, atherosclerotic changes of ASA and KA can lead to dissection or aneurysmal dilatation. In a review of 32 cases of RAA associated with KA and ASA reported from 1966 through 2002, 12 were associated with aortic dissection, and 2 included aneurysmal rupture. ${ }^{1}$ Therefore a precise anatomic delineation of RAA is important for appropriate therapy.

TEE, MSCT, and magnetic resonance imaging are highly accurate in evaluating aortic dissection, and catheter angiography is now used primarily for treating complications. ${ }^{2,3}$ TEE has been reported to be the diagnostic imaging modality of choice in the unstable patient because it can detect ascending aortic dissection, which necessitates urgent surgical intervention. ${ }^{3}$ Intraoperative TEE has also been recommended as a primary diagnostic modality to rule out aortic dissection. ${ }^{4}$ However, it should be cautiously applied in assessing RAA because of the greater potential for unfamiliarity of the examiner with such complicated anatomy. ${ }^{5}$ Furthermore, TEE examination of RAA is easily interfered with by the trachea, which lies between the esophagus and the ascending aorta. In the present case TEE not only failed to identify the RAA and the ascending aorta but also misinterpreted the KA dissection as an aortic arch dissection. On the contrary, MSCT with multiplanar reconstruction offered a comprehensive anatomic delineation of the RAA, KA, and ALSA. Most importantly, it demonstrated the entrance tear of the dissection at the KA, showed the exact extent of the dissection flaps, and excluded the involvement
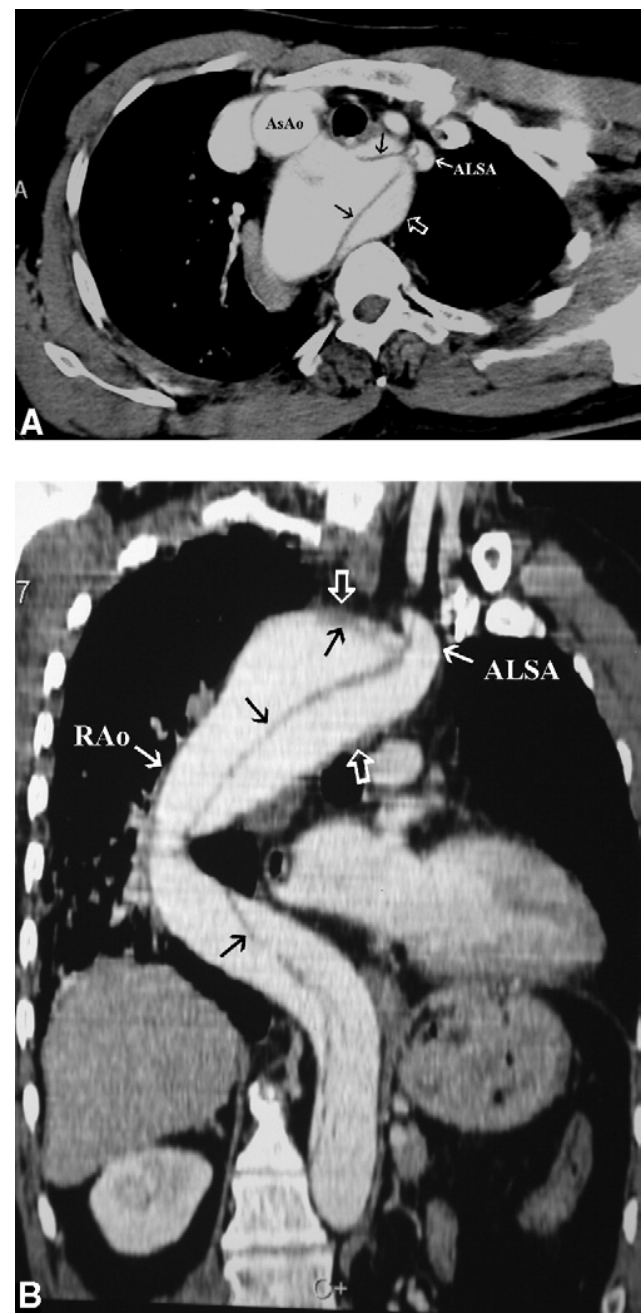

Figure 2. A, Axial contrast-enhanced computed tomography shows a Kommerell's aneurysm (open arrow) with an intimal flap (arrows) extending to the aberrant left subclavian artery (ALSA). Note that the right ascending aorta $(A s A o)$ is not affected. $B$, Oblique coronal reconstruction view shows the dissection (arrows) of the Kommerell's aneurysm (open arrows) involving the ALSA, right descending thoracic aorta (RAo), and abdominal aorta. Note that the aortic arch is spared.

of the aortic arch and ascending aorta. These findings established the definitive diagnosis of type B dissection. Instead of immediate surgical intervention, the patient was successfully managed with conservative treatment. Although the patient refused elective surgical resection of the KA, he remained in good condition during 3 years of follow-up.

In summary, echocardiographic findings of RAA and KA can lead to confusion. MSCT with multiplanar reconstruction can allow comprehensive elucidation of the congenital arch anomaly associated with acute aortic diseases, facilitating appropriate treatment. 


\section{References}

1. Cina CS, Althani H, Pasenau J, Abouzahr L. Kommerell's diverticulum and right-sided aortic arch: a cohort study and review of the literature. J Vasc Surg. 2004:39:131-9.

2. Kapustin AJ, Litt HI. Diagnostic imaging for aortic dissection. Semin Thorac Cardiovasc Surg. 2005;17:214-23.

3. Flachskampf FA. Assessment of aortic dissection and hematoma. Semin Cardiothorac Vasc Anesth. 2006;10:83-8.
4. Eltzschig HK, Rosenberger P, Lekowski RW Jr, Scott JD, Locke A, Shekar PS, et al. Role of transesophageal echocardiography in patients with suspected aortic dissection. J Am Soc Echocardiogr. 2005;18: 1221.

5. Ko SF, Ng SH, Fu M, Lo PH, Cheng YF, Lee TY. Dissection of retroesophageal aortic diverticulum and descending aorta in a patient with right aortic arch: magnetic resonance demonstration. Cardiovasc Intervent Radiol. 1996;19:438-41.

\title{
Cardiac pheochromocytoma: A new case reported
}

\author{
Gustavo Knop, MD, ${ }^{a}$ and Roberto Margaria, MD, ${ }^{\mathrm{b}}$ La Pampa, Argentina
}

$\mathrm{P}$ rimary cardiac pheochromocytomas are exceptional. Less than $1 \%$ of these tumors have a thoracic location, being the rarest cardiac occurrence. Thus far, about 50 cases have been reported in the world. We present a case of an intrapericardial tumor that turned out to be a pheochromocytoma in a middle-aged woman.

\section{Clinical Summary}

A 38-year-old woman was referred to a cardiologist at the local hospital because of severe hypertension, paroxysmal episodes of palpitations, headaches, profuse perspiration, and a reddish face.

A transthoracic echocardiogram was performed, and an intrapericardial mass was observed. A coronary angiogram was done and showed the presence of a highly vascularized mass with arterial supply by the circumflex artery (Figure 1). Magnetic resonance imaging confirmed a solid tumor in direct contact with the left atrium and the left superior pulmonary vein. A transesophageal echocardiogram confirmed the location of the mass.

The patient was referred to us for surgical resection of the intrapericardial tumor. At this point, the fact that this tumor was so vascularized raised our clinical suspicion that we might be dealing with a catecholamine-secreting tumor. Laboratory findings of 1100 pg-mL of urine norepinephrine and very high levels of vanillylmandelic acid confirmed that the patient had a pheochromocytoma. Therefore consequently, in the next step of the investigation, we ruled out the classical abdominal location because all the abdominal image studies were negative. A metaiodobenzylguanidine scan study was performed, and the result was not conclusive.

\footnotetext{
From the Department of Cardiovascular Surgery, Argentina Clinic, General Pico, La Pampa, Argentina.

Received for publication June 25, 2006; accepted for publication July 12 , 2006.

Address for reprints: Gustavo Knop, MD, Clinica Argentina, Cirugia Cardiovascular, Av San Martin 730, General Pico, La Pampa 6300, Argentina (E-mail: drknop@hotmail.com)

J Thorac Cardiovasc Surg 2006;132:1230-1

$0022-5223 / \$ 32.00$

Copyright (C) 2006 by The American Association for Thoracic Surgery doi:10.1016/j.jtcvs.2006.07.028
}

A bibliographic revision of cardiac tumors increased our conviction that a car-

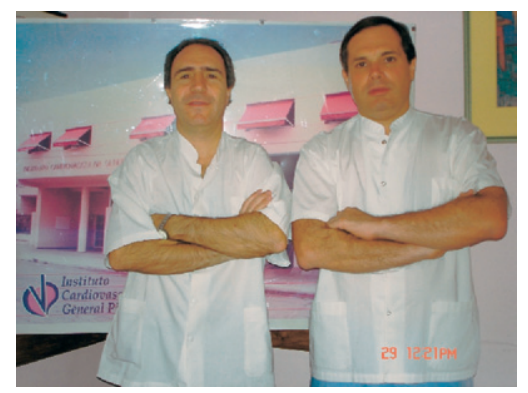

Drs Knop and Margaria (left to right) diac pheochromocytoma, although an extremely rare condition, was present.

After appropriate preoperative preparation of the patient with $\alpha$ - and $\beta$-adrenergic blockers, the operation was performed during cardiopulmonary bypass with bicaval cannulation. Any manipulation of the heart was avoided. The aorta was crossclamped, antegrade cardioplegia was administered, the superior and inferior venae cavae were snared, the right atrium was opened, and the blood and cardioplegia fluid returning from the coronary sinus were sucked and discarded to avoid possible incorporation into the pump circuit of catecholamines released by the tumor and the concomitant occurrence of massive peripheral vasoconstriction. After this precautionary maneuver, our attention was turned to the tumor. A solid and highly vascularized mass 4 by $4 \mathrm{~cm}$ in size (Figure 2) was present on the roof of the left atrium, without a cleavage plane with the left atrial wall and very dense adhesions to the left superior pulmonary vein. The feeding artery of the tumor originating in the circumflex was identified and ligated during the

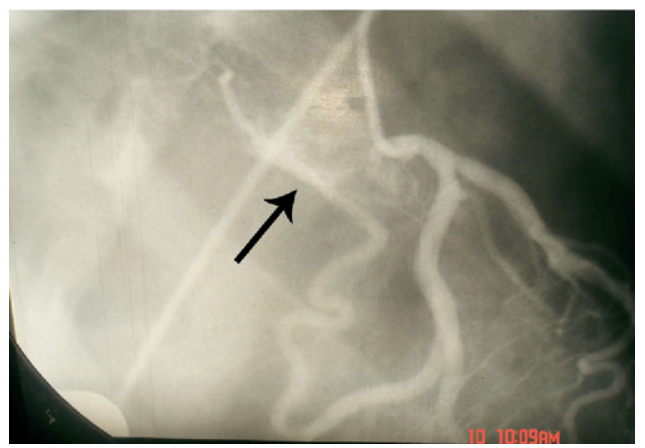

Figure 1. Coronary angiogram showing the arterial supply of the tumor by a significant branch of the circumflex artery and the highly vascularized nature of the catecholamine-secreting tumor. 